Pacific Journal of Mathematics

OPERATOR-VALUED FEYNMAN INTEGRALS VIA 


\section{OPERATOR-VALUED FEYNMAN INTEGRALS VIA CONDITIONAL FEYNMAN INTEGRALS}

\section{Dong Myung Chung, Chull Park, and David Skoug}

In this paper we use the concept of the conditional Feynman integral to obtain the analytic operator-valued Feynman integral of various functions.

1. Introduction. In [1] Cameron and Storvick introduced a very general analytic operator-valued function space "Feynman integral", $J_{q}^{\text {an }}(F)$, which mapped an $L_{2}\left(\mathbb{R}^{\nu}\right)$ function $\psi$ into an $L_{2}\left(\mathbb{R}^{\nu}\right)$ function $\left(J_{q}^{\text {an }}(F) \psi\right)(\vec{\xi})$. Further work involving the $L_{2} \rightarrow L_{2}$ theory includes $[2,3,16-18]$. In $[4,19]$ the existence of the Feynman integral as an operator from $L_{1}(\mathbb{R})$ to $L_{\infty}(\mathbb{R})$ was studied. Finally in [20], an $L_{p} \rightarrow L_{p^{\prime}}$ theory, $1 / p+1 / p^{\prime}=1$, was developed for $1<p \leq 2$. Related stability results were established in [10, 25].

In [15], Chung and Skoug introduced the concept of a conditional Feynman integral. In this paper we further develop this concept and proceed to express operator-valued Feynman integrals in terms of conditional Feynman integrals. In particular we show that various operator-valued Feynman integrals can be obtained using the formula

$$
\begin{aligned}
& \left(J_{q}^{\mathrm{an}}(F) \psi\right)(\vec{\xi})=\int_{\mathbb{R}^{\nu}} E^{\operatorname{anf}_{q}}(F \mid X)(\vec{\xi})(\vec{\eta})\left[\frac{q}{2 \pi i T}\right]^{\nu / 2} \\
& \cdot \exp \left\{\frac{q i}{2 T}\|\vec{\eta}-\vec{\xi}\|^{2}\right\} \psi(\vec{\eta}) d \vec{\eta}
\end{aligned}
$$

where $E^{\operatorname{anf}_{q}}(F \mid X)$ is the conditional analytic Feynman integral of $F$ given $X$. Thus $J_{q}^{\text {an }}(F)$ can be interpreted as an integral operator with kernel

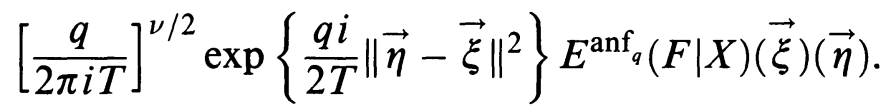

In [5], Cameron and Storvick introduced a Banach algebra $S(\nu)$ of functions on Wiener space which are a kind of stochastic Fourier transform of Borel measures on $L_{2}^{\nu}[0, T]$. In $\S 3$ of this paper we show that for all $F$ in $S(\nu), J_{q}^{\text {an }}(F)$ is given by (1.1) and can be 
interpreted as a bounded linear operator from $L_{1}\left(\mathbb{R}^{\nu}\right)$ to $L_{\infty}\left(\mathbb{R}^{\nu}\right)$. In this setting we also obtain some stability results.

A very important class of functions in Quantum Mechanics are functions on Wiener space $C_{0}^{\nu}[0, T]$ of the form

$$
F(\vec{x})=\exp \left\{\int_{0}^{T} \theta(s, \vec{x}(s)) d s\right\}
$$

where $\theta:[0, T] \times \mathbb{R}^{\nu} \rightarrow \mathbb{C}$. In $\S \S 4$ and 5 , using a useful series expansion formula, we show that for appropriate $\theta, J_{q}^{\text {an }}(F)$ exists as an operator from $L_{1}$ to $L_{\infty}$ and is given by (1.1).

2. Definitions and preliminaries. Let $\nu$ be a positive integer. Let $C^{\nu}[0, T]$ denote the space of $\mathbb{R}^{\nu}$-valued continuous functions on $[0, T]$ and let $C_{0}^{\nu}[0, T]$ denote $\nu$-dimensional Wiener space; that is the set of all functions $\vec{x}(t)$ in $C^{\nu}[0, T]$ such that $\vec{x}(0)=\overrightarrow{0}$. Let $\mathscr{M}$ denote the class of all Wiener measurable subsets of $C_{0}^{\nu}[0, T]$ and let $m$ denote $\nu$-dimensional Wiener measure. $\left(C_{0}^{\nu}[0, T], \mathscr{M}, m\right)$ is a complete measure space and we denote the Wiener integral of a Wiener measurable function $F$ by

$$
\int_{C_{0}^{\nu}} F(\vec{x}) m(d \vec{x})
$$

whenever the integral exists.

A set $E \in \mathscr{M}$ is said to be scale-invariant measurable [11, 21] provided $\rho E \in \mathscr{M}$ for each $\rho>0$ and a scale-invariant measurable set $N$ is said to be scale-invariant null provided $m(\rho N)=0$ for each $\rho>0$. A property which holds except on a scale-invariant null set is said to hold scale-invariant almost everywhere (s-a.e.).

Next we give Yeh's definition of the conditional Wiener integral [29].

Definition 1. Let $X$ be an $\mathbb{R}^{\nu}$-valued Wiener measurable function on $C_{0}^{\nu}[0, T]$ and let $F$ be a complex-valued Wiener integral on $C_{0}^{\nu}[0, T]$. Let $P_{X}$ be the probability distribution of $X$, i.e., for all $B \in \mathscr{B}^{\nu}$, the Borel sets in $\mathbb{R}^{\nu}, P_{X}(B)=m\left(X^{-1}(B)\right)$. The conditional Wiener integral of $F$ given $X$ is by definition the equivalence class of Borel measurable and $P_{X}$-integrable functions $\phi$ on $\mathbb{R}^{\nu}$, modulo null functions on $\left(\mathbb{R}^{\nu}, \mathscr{B}^{\nu}, P_{X}\right)$, such that for all $B \in \mathscr{B}^{\nu}$,

$$
\int_{X^{-1}(B)} F(\vec{x}) m(d \vec{x})=\int_{B} \phi(\vec{\eta}) P_{X}(d \vec{\phi})
$$


By the Radon-Nikodym Theorem such a function $\phi$ exists and is determined up to a null function on $\left(\mathbb{R}^{\nu}, \mathscr{B}^{\nu}, P_{X}\right)$. We let $E(F \mid X)$ denote a representative of the equivalence class and so for all $B \in \mathscr{B}^{\nu}$,

$$
\int_{X^{-1}(B)} F(\vec{x}) m(d \vec{x})=\int_{B} E(F \mid X)(\vec{\eta}) P_{X}(d \vec{\eta}) .
$$

REMARK. In [27], Park and Skoug showed that if $F$ is Borel measurable and Wiener integrable and if $X(\vec{x})=\vec{x}(T)$, then the conditional Wiener integral $E(F \mid X)$ can be expressed in terms of an ordinary Wiener integral by the formula

$$
E(F \mid X)(\vec{\eta})=\int_{C_{0}^{\nu}} F\left(\vec{x}(\cdot)-\frac{\dot{T}}{T}(T)+\frac{\dot{T}}{T}\right) m(d \vec{x}) .
$$

We are now ready to define the conditional analytic Feynman integral of a function $F$ given $X$.

Definition 2. Let $\mathbb{C}, \mathbb{C}_{+}$and $\mathbb{C}_{+}^{\sim}$ denote respectively the complex numbers, the complex numbers with positive real part, and the nonzero complex numbers with nonnegative real part. Let $F$ : $C^{\nu}[0, T] \rightarrow \mathbb{C}$ be such that for each $\lambda>0$,

$$
\int_{C_{0}^{\nu}}\left|F\left(\lambda^{-1 / 2} \vec{x}+\vec{\xi}\right)\right| m(d \vec{x})<\infty
$$

for a.e. $\vec{\xi} \in \mathbb{R}^{\nu}$. Let $X: C^{\nu}[0, T] \rightarrow \mathbb{R}^{\nu}$ be such that for each $\lambda>0$ and a.e. $\vec{\xi} \in \mathbb{R}^{\nu}, X\left(\lambda^{-1 / 2} \vec{x}+\vec{\xi}\right)$ is a Wiener measurable function of $\vec{x}$ on $C_{0}^{\nu}[0, T]$; i.e., for a.e. $\vec{\xi}$ in $\mathbb{R}^{\nu}, Y(\vec{x}) \equiv X\left(\lambda^{-1 / 2} \vec{x}+\vec{\xi}\right)$ is scale-invariant measurable on $C_{0}^{\nu}[0, T]$. For $\lambda>0$ and $\vec{\xi} \in \mathbb{R}^{\nu}$, let

$$
J_{\lambda}(\vec{\xi}, \vec{\eta}) \equiv E\left(F\left(\lambda^{-1 / 2} \vec{x}+\vec{\xi}\right) \mid X\left(\lambda^{-1 / 2} \vec{x}+\vec{\xi}\right)\right)(\vec{\eta})
$$

denote the conditional Wiener integral of $F\left(\lambda^{-1 / 2} \vec{x}+\vec{\xi}\right)$ given $X\left(\lambda^{-1 / 2} \vec{x}+\vec{\xi}\right)$. If for a.e. $\vec{\eta} \in \mathbb{R}^{\nu}$, there exists a function $J_{\lambda}^{*}(\vec{\xi}, \vec{\eta})$, analytic in $\lambda$ on $\mathbb{C}_{+}$such that $J_{\lambda}^{*}(\vec{\xi}, \vec{\eta})=J_{\lambda}(\vec{\xi}, \vec{\eta})$ for all $\lambda>0$, then $J_{\lambda}^{*}(\vec{\xi}, \cdot)$ is defined to be the conditional Wiener integral of $F$ given $X$ with parameter $\lambda$ and we write

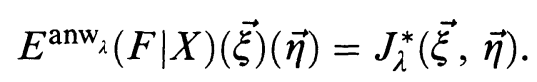

If for fixed real $q \neq 0$, the limit

$$
\lim _{\lambda \rightarrow-i q} E^{\mathrm{anw}_{\lambda}}(F \mid X)(\vec{\xi})(\vec{\eta})
$$


exists for a.e. $\vec{\eta} \in \mathbb{R}^{\nu}$ where $\lambda \rightarrow-i q$ through $\mathbb{C}_{+}$, we will denote

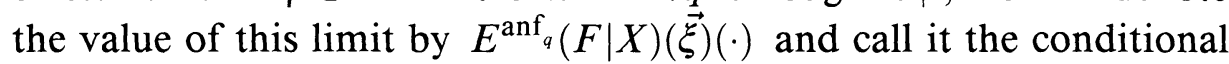
analytic Feynman integral of $F$ given $X$ with parameter $q$.

We finish this section by stating the definition of the analytic operator-valued Feynman integral as an element of $\mathscr{L}\left(L_{1}\left(\mathbb{R}^{\nu}\right), L_{\infty}\left(\mathbb{R}^{\nu}\right)\right)$.

Definition 3. Let $F: C^{\nu}[0, T] \rightarrow \mathbb{C}$. Given $\lambda>0, \psi$ in $L_{1}\left(\mathbb{R}^{\nu}\right)$ and $\vec{\xi}$ in $\mathbb{R}^{\nu}$, let

$$
\left(I_{\lambda}(F) \psi\right)(\vec{\xi}) \equiv \int_{C_{0}^{\nu}} F\left(\lambda^{-1 / 2} \vec{x}+\vec{\xi}\right) \psi\left(\lambda^{-1 / 2} \vec{x}(T)+\vec{\xi}\right) m(d \vec{x}) .
$$

If $I_{\lambda}(F) \psi$ is in $L_{1}\left(\mathbb{R}^{\nu}\right)$ as a function of $\vec{\xi}$ and if the correspondence $\psi \rightarrow I_{\lambda}(F) \psi$ gives an element of $\mathscr{L}\left(L_{1}\left(\mathbb{R}^{\nu}\right), L_{\infty}\left(\mathbb{R}^{\nu}\right)\right)$, the space of continuous linear operators from $L_{1}\left(\mathbb{R}^{\nu}\right)$ to $L_{\infty}\left(\mathbb{R}^{\nu}\right)$, we say that the operator-valued function space integral $I_{\lambda}(F)$ exists. Next suppose there exists an $\mathscr{L}$-valued function which is analytic in $\mathbb{C}_{+}$and agrees with $I_{\lambda}(F)$ on $(0, \infty)$; then this $\mathscr{L}$-valued function is denoted by $I_{\lambda}^{\mathrm{an}}(F)$ and is called the analytic operator-valued Wiener integral of $F$ associated with $\lambda$. Finally, for $\lambda=-i q \in \mathbb{C}_{+}$, suppose there exists an operator $J_{q}^{\text {an }}(F)$ in $\mathscr{L}\left(L_{1}\left(\mathbb{C}^{\nu}\right), L_{\infty}\left(\mathbb{R}^{\nu}\right)\right)$ such that for every $\psi$ in $L_{1}\left(\mathbb{R}^{\nu}\right)$,

$$
\left\|J_{q}^{\mathrm{an}}(F) \psi-I_{\lambda}^{\mathrm{an}}(F) \psi\right\|_{\infty} \rightarrow 0
$$

as $\lambda \rightarrow-i q$ through $\mathbb{C}_{+}$; then $J_{q}^{\text {an }}(F)$ is called the analytic operatorvalued Feynman integral of $F$ with parameter $q$.

Finally we state the following well-known integration formula

$$
\begin{aligned}
& \int_{\mathbb{R}^{\nu}} \exp \left\{-\frac{b}{2}\|\vec{\eta}\|^{2}+i\langle\vec{\eta}, \vec{\xi}\rangle\right\} d \vec{\eta} \\
&=\left[\frac{2 \pi}{b}\right]^{\nu / 2} \exp \left\{-\frac{1}{2 b}\|\vec{\xi}\|^{2}\right\}, \quad \operatorname{Re} b>0
\end{aligned}
$$

which we use several times in this paper.

3. The $S(\nu)$ theory. In [5] Cameron and Storvick introduced a Banach algebra $S(\nu)$ of functions on $\nu$-dimensional Wiener space each of which is a type of a stochastic Fourier transform of bounded $\mathbb{C}$-valued Borel measures. They showed that the analytic (but scalarvalued) Feynman integral exists for all elements of $S(\nu)$. Further work on $S(\nu)$ includes $[7,8,13,22,23,24]$. 
The Banach algebra $S(\nu)$ consists of functions on $C_{0}^{\nu}[0, T]$ expressible in the form

$$
F(\vec{x})=\int_{L_{2}^{\nu}[0, T]} \exp \left\{i \sum_{j=1}^{\nu} \int_{0}^{T} v_{j}(s) \tilde{d} x_{j}(s)\right\} d \sigma(\vec{v})
$$

for $s$-a.e. $\vec{x}=\left(x_{1}, \ldots, x_{\nu}\right)$ in $C_{0}^{\nu}[0, T]$ where $\sigma$ is an element of $M\left(L_{2}^{\nu}[0, T]\right)$, the space of $\mathbb{C}$-valued, countably additive Borel measures on $L_{2}^{\nu}[0, T]$ and the integrals $\int_{0}^{T} v_{j}(s) \tilde{d} x_{j}(s)$ are Paley-WienerZygmund (P.W.Z.) stochastic integrals [23, p. 280].

REMARK. If $F$ is in $S(\nu)$ then $F$ is scale-invariant measurable and $s$-a.e. defined on $C_{0}^{\nu}[0, T]$. Furthermore there is a natural way of regarding $F$ as defined on $C^{\nu}[0, T]:$ If $\vec{x}$ in $C_{0}^{\nu}[0, T]$ is such that $F(\vec{x})$ is defined, then by $(3.1), F(\vec{x}+\vec{\xi})=F(\vec{x})$ for all $\vec{\xi} \in \mathbb{R}^{\nu}$.

First, for $F$ in $S(\nu)$ and $X(\vec{y})=\vec{y}(T)$, we obtain a formula for

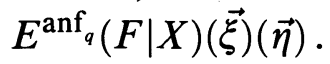

THEOREM 3.1. Let $F \in S(\nu)$ be given by (3.1) and let $X: C^{\nu}[0, T]$ $\rightarrow \mathbb{R}^{\nu}$ be given by $X(\vec{y})=\vec{y}(T)$. Then for all $(\vec{\xi}, \vec{\eta}) \in \mathbb{R}^{\nu} \times \mathbb{R}^{\nu}$

$$
\begin{aligned}
E^{\text {anw }_{\lambda}(F \mid X)(\vec{\xi})(\vec{\eta})} & =\int_{L_{2}^{\nu}[0, T]} \exp \left\{-\frac{1}{2 \lambda T} \sum_{j=1}^{\nu}[\right. \\
& \left.+\frac{i}{T}\langle\vec{\eta}-\vec{\xi}, \vec{B}\rangle\right\} d \sigma(\vec{v})
\end{aligned}
$$

for all $\lambda \in \mathbb{C}_{+}$and

$$
\begin{aligned}
E^{\operatorname{anf}_{q}(F \mid X)(\vec{\xi})(\vec{\eta})} & =\int_{L_{2}^{\nu}[0, t]} \exp \left\{-\frac{i}{2 q T} \sum_{j=1}^{\nu}[\right. \\
& \left.+\frac{i}{T}\langle\vec{\eta}-\vec{\xi}, \vec{B}\rangle\right\} d \sigma(\vec{v})
\end{aligned}
$$

for all real $q \neq 0$ where

$$
\vec{B}=\left(b_{1}, \ldots, b_{\nu}\right)=\left(\int_{0}^{T} v_{1}(s) d s, \ldots, \int_{0}^{T} v_{\nu}(s) d s\right) .
$$


Proof. Using (3.1), (2.2), the Fubini Theorem, (3.4) and a fundamental Wiener integration formula involving P.W.Z. integrals, for all $\lambda>0$ and all $(\vec{\xi}, \vec{\eta}) \in \mathbb{R}^{\nu} \times \mathbb{R}^{\nu}$ we obtain the formula

$$
\begin{aligned}
& E\left(F\left(\lambda^{-1 / 2} \vec{x}+\vec{\xi}\right) \mid X\left(\lambda^{-1 / 2} \vec{x}+\vec{\xi}\right)\right)(\vec{\eta}) \\
& =\int_{C_{0}^{\nu}}\left[\int _ { L _ { 2 } ^ { \nu } [ 0 , T ] } \operatorname { e x p } \left\{i \sum _ { j = 1 } ^ { \nu } \int _ { 0 } ^ { T } v _ { j } ( s ) \tilde { d } \left[\lambda^{-1 / 2} x_{j}(s)-\lambda^{-1 / 2} \frac{s}{T} x_{j}(T)\right.\right.\right. \\
& \left.\left.\left.+\frac{s}{T}\left(\eta_{j}-\xi_{j}\right)\right]\right\} C_{0}^{\nu} d \sigma(\vec{v})\right] m(d \vec{x}) \\
& =\int_{L_{2}^{\nu}[0, T]}\left[\int _ { C _ { 0 } ^ { \nu } } \operatorname { e x p } \left\{\frac { i } { \sqrt { \lambda } } \sum _ { j = 1 } ^ { \nu } \left[\int_{0}^{T} v_{j}(s) \tilde{d} x_{j}(s)\right.\right.\right. \\
& \left.-\frac{x_{j}(T)}{T} \int_{0}^{T} v_{j}(s) d s\right] \\
& \left.\left.+\frac{i}{T} \sum_{j=1}^{\nu}\left(\eta_{j}-\xi_{j}\right) \int_{0}^{T} v_{j}(s) d s\right\} m(d \vec{x})\right] d \sigma(\vec{v}) \\
& =\int_{L_{2}^{\nu}[0, T]} \exp \left\{\frac{i}{T}\langle\vec{\eta}-\vec{\xi}, \vec{B}\rangle\right\} \\
& \cdot \int_{C_{0}^{\nu}} \exp \left\{\frac{i}{\sqrt{\lambda}} \sum_{j=1}^{\nu} \int_{0}^{T}\left[v_{j}(s)-\frac{b_{j}}{T}\right] \tilde{d} x_{j}(s)\right\} m(d \vec{x}) d \sigma(\vec{v}) \\
& =\int_{L_{2}^{\nu}[0, T]} \exp \left\{\frac{i}{T}\langle\vec{\eta}-\vec{\xi}, \vec{B}\rangle\right\} \\
& \cdot \exp \left\{-\frac{1}{2 \lambda} \sum_{j=1}^{\nu} \int_{0}^{T}\left[v_{j}(s)-\frac{b_{j}}{T}\right]^{2} d s\right\} d \sigma(\vec{v}) \\
& =\int_{L_{2}^{\nu}[0, T]} \exp \left\{-\frac{1}{2 \lambda T} \sum_{j=1}^{\nu}\left[T\left\|v_{j}\right\|^{2}-b_{j}^{2}\right]+\frac{i}{T}\langle\vec{\eta}-\vec{\xi}, \vec{B}\rangle\right\} d \sigma(\vec{v}) .
\end{aligned}
$$

Using the Cauchy-Schwarz inequality we see that

$$
b_{j}^{2}=\left[\int_{0}^{T} v_{j}(s) d s\right]^{2} \leq \int_{0}^{T} 1^{2} d s \int_{0}^{T} v_{j}^{2}(s) d s=T\left\|v_{j}\right\|^{2} .
$$

Thus, since $\sigma \in M\left(L_{2}^{\nu}[0, T]\right)$, the last expression on the right-hand side of (3.5) is an analytic function of $\lambda$ throughout $\mathbb{C}_{+}$and is a 
continuous function of $\lambda$ for $\lambda \in \mathbb{C}_{+}^{\sim}$. Thus (see Definition 2 in $\S 2$ above) equations (3.2) and (3.3) are established.

Theorem 3.2. Let $F$ and $X$ be as in Theorem 3.1. Then for all real $q \neq 0$, the analytic operator-valued Feynman integral $J_{q}^{\text {an }}(F)$ exists as an element of $\mathscr{L}\left(L_{1}\left(\mathbb{R}^{\nu}\right), L_{\infty}\left(\mathbb{R}^{\nu}\right)\right)$ and for each $\psi \in L_{1}\left(\mathbb{R}^{\nu}\right)$ we have

$$
\begin{aligned}
& \left(J_{q}^{\mathrm{an}}(F) \psi\right)(\vec{\xi})
\end{aligned}
$$

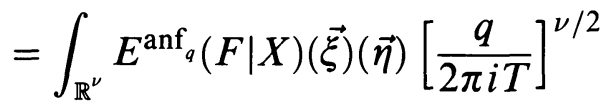

$$
\begin{aligned}
& \cdot \exp \left\{\frac{i q\|\vec{\eta}-\vec{\xi}\|^{2}}{2 T}\right\} \psi(\vec{\eta}) d \vec{\eta}
\end{aligned}
$$

for all $\vec{\xi} \in \mathbb{R}^{\nu}$.

Proof. Let $\psi \in L_{1}\left(\mathbb{R}^{\nu}\right)$ be given. We can assume that $\psi$ is Borel measurable since if $\psi$ is only Lebesgue measurable then there exists a Borel measurable function $\psi_{1}$ such that $\psi_{1}=\psi$ a.e. on $\mathbb{R}^{\nu}$. Moreover $\psi_{1}$ is unique up to Borel null sets. But $F$ is also Borel measurable and so using equation (2.2) it is quite easy to see that

$$
\begin{gathered}
E\left(F\left(\lambda^{-1 / 2} \vec{x}+\vec{\xi}\right) \psi\left(\lambda^{-1 / 2} \vec{x}(T)+\vec{\xi}\right) \mid X\left(\lambda^{-1 / 2} \vec{x}+\vec{\xi}\right)\right)(\vec{\eta}) \\
=\psi(\vec{\eta}) E\left(F\left(\lambda^{-1 / 2} \vec{x}+\vec{\xi}\right) \mid X\left(\lambda^{-1 / 2} \vec{x}+\vec{\xi}\right)\right)(\vec{\eta}) .
\end{gathered}
$$

Then by the definition of $I_{\lambda}(F) \psi$ and equation (2.1) it follows that

$$
\begin{aligned}
\left(I_{\lambda}(F) \psi(\vec{\xi})=\int_{C_{0}^{\nu}} F\left(\lambda^{-1 / 2} \vec{x}+\vec{\xi}\right) \psi\left(\lambda^{-1 / 2} \vec{x}(T)+\vec{\xi}\right) m(d \vec{x})\right. \\
\left.=\int_{\mathbb{R}^{\nu}} E\left(F\left(\lambda^{-1 / 2} \vec{x}+\vec{\xi}\right) \psi\left(\lambda^{-1 / 2} \vec{x}(T)+\vec{\xi}\right) \mid X^{-1 / 2} \vec{x}+\vec{\xi}\right)\right)(\vec{\eta}) \\
\cdot\left[\frac{\lambda}{2 \pi T}\right]^{\nu / 2} \exp \left\{-\frac{\lambda}{2 T}\|\vec{\eta}-\vec{\xi}\|^{2}\right\} d \vec{\eta} \\
=\int_{\mathbb{R}^{\nu}} E\left(F\left(\lambda^{-1 / 2} \vec{x}+\vec{\xi}\right) \mid X\left(\lambda^{-1 / 2} \vec{x}+\vec{\xi}\right)\right)(\vec{\eta})\left[\frac{\lambda}{2 \pi T}\right]^{\nu / 2} \\
\cdot \exp \left\{-\frac{\lambda}{2 T}\|\vec{\eta}-\vec{\xi}\|^{2}\right\} \psi(\vec{\eta}) d \vec{\eta}
\end{aligned}
$$

for all $\lambda>0$. Then, using Theorem 3.1 and Morera's Theorem, we 
obtain that

$$
\begin{aligned}
& \left(I_{\lambda}^{\mathrm{an}}(F) \psi\right)(\vec{\xi}) \\
& =\int_{\mathbb{R}^{\nu}} E^{\mathrm{anw}_{\lambda}}(F \mid X)(\vec{\xi})(\vec{\eta})\left[\frac{\lambda}{2 \pi T}\right]^{\nu / 2} \\
& \quad \cdot \exp \left\{-\frac{\lambda\|\vec{\eta}-\vec{\xi}\|^{2}}{2 T}\right\} \psi(\vec{\eta}) d \vec{\eta}
\end{aligned}
$$

for all $\lambda \in \mathbb{C}_{+}$and all $\vec{\xi} \in \mathbb{R}^{\nu}$.

But since $E^{\operatorname{anw}_{\lambda}(F \mid X)(\vec{\xi})(\vec{\eta})}$ is bounded and $\psi \in l_{1}\left(\mathbb{R}^{\nu}\right)$, we see that the right-hand side of $(3.7)$ is continuous in $\lambda$ on $\mathbb{C}_{+}^{\sim}$. Thus

$$
\begin{aligned}
& \lim _{\lambda \rightarrow-i q}\left(I_{\lambda}^{\mathrm{an}}(F) \psi\right)(\vec{\xi})=\int_{\mathbb{R}^{\nu}} E^{\operatorname{anf}_{q}}(F \mid X)(\vec{\xi}, \vec{\eta})\left[\frac{q}{2 \pi i T}\right]^{\nu / 2} \\
& \cdot \exp \left\{\frac{i q\|\vec{\eta}-\vec{\xi}\|^{2}}{2 T}\right\} \psi(\vec{\eta}) d \vec{\eta}
\end{aligned}
$$

for each $\vec{\xi} \in \mathbb{R}^{\nu}$. Thus $J_{q}^{\text {an }}(F)$ exists as an element of

$$
\mathscr{L}\left(L_{1}\left(\mathbb{R}^{\nu}\right), L_{\infty}\left(\mathbb{R}^{\nu}\right)\right)
$$

and (3.6) is established.

The following stability results follow quite readily using equations (3.3) and (3.6).

THEOREM 3.3. Let $\left\{\sigma_{n}\right\}$ be a sequence of elements from $M\left(L_{2}^{\nu}[0, T]\right)$ that converge weakly to $\sigma \in M\left(L_{2}^{\nu}[0, T]\right)$, let $F$ be given by (3.1) and for $n=1,2, \ldots$, let

$$
F_{n}(\vec{x})=\int_{L_{2}^{\nu}[0, T]} \exp \left\{i \sum_{j=1}^{\nu} \int_{0}^{T} v_{j}(s) \tilde{d} x(s)\right\} d \sigma_{n}(\vec{v})
$$

for s-a.e. $\vec{x} \in C_{0}^{\nu}[0, T]$. Let $\left\{q_{n}\right\}$ be a sequence of real numbers converging to $q \neq 0$ and let $\left\{\psi_{n}\right\}$ be a sequence from $L_{1}\left(\mathbb{R}^{\nu}\right)$ converging in $L_{1}$-norm to $\psi \in L_{1}\left(\mathbb{R}^{\nu}\right)$. Then as $n \rightarrow \infty$ :

$$
E^{\operatorname{anf}_{q}}\left(F_{n} \mid X\right)(\vec{\xi})(\vec{\eta}) \rightarrow E^{\operatorname{anf}_{q}(F \mid X)(\vec{\xi})(\vec{\eta})}
$$

for all $(\vec{\xi}, \vec{\eta}) \in \mathbb{R}^{\nu} \times \mathbb{R}^{\nu}$, 


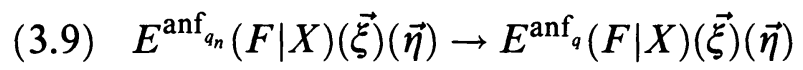

for all $(\vec{\xi}, \vec{\eta}) \in \mathbb{R}^{\nu} \times \mathbf{R}^{\nu}$, $J_{q}^{\text {an }}\left(F_{n}\right) \psi \rightarrow J_{q}^{\text {an }}(F) \psi \quad$ in $L_{\infty}$-norm on $\mathbb{R}^{\nu}$, $J_{q_{n}}^{\mathrm{an}}(F) \psi \rightarrow J_{q}^{\mathrm{an}}(F) \psi \quad$ in $L_{\infty}$-norm on $\mathbb{R}^{\nu}, \quad$ and $J_{q}^{\mathrm{an}}(F) \psi_{n} \rightarrow J_{q}^{\mathrm{an}}(F) \psi \quad$ in $L_{\infty}$-norm on $\mathbb{R}^{\nu}$.

4. A useful series expansion. In this section for $F$ given by (1.2) with minimal conditions on $\theta$ and $X(\vec{y})=\vec{y}(T)$ we obtain a useful series expansion for $E\left(F\left(\lambda^{-1 / 2} \vec{x}+\vec{\xi}\right) \mid X\left(\lambda^{-1 / 2} \vec{x}+\vec{\xi}\right)\right)(\vec{\eta})$.

THEOREM 4.1. Let $F(\vec{x})$ be given by (1.2) where $\theta$ is Borel measurable and where for each $\lambda>0$

$$
\int_{C_{0}^{\nu}}\left|F\left(\lambda^{-1 / 2} \vec{x}+\vec{\xi}\right)\right| m(d \vec{x})<\infty
$$

for a.e. $\vec{\xi} \in \mathbb{R}^{\nu}$. Then for each $\lambda>0$

$$
\begin{aligned}
& E\left(F\left(\lambda^{-1 / 2} \vec{x}+\vec{\xi}\right) \mid X\left(\lambda^{-1 / 2} \vec{x}+\vec{\xi}\right)\right)(\vec{\eta})\left[\frac{\lambda}{2 \pi T}\right]^{\nu / 2} \\
& \quad \cdot \exp \left\{-\frac{\lambda}{2 T}\|\vec{\eta}-\vec{\xi}\|^{2}\right\} \\
& =\sum_{n=0}^{\infty} \int_{\Delta_{n}(T)}\left[\frac{\lambda^{n+1}}{(2 \pi)^{n+1} s_{1}\left(s_{2}-s_{1}\right) \cdots\left(s_{n}-s_{n-1}\right)\left(T-s_{n}\right)}\right]^{\nu / 2} \\
& \quad \int_{\mathbb{R}^{n \nu}}\left[\prod_{j=1}^{n} \theta\left(s_{j}, \vec{w}_{j}\right)\right] \\
& \quad \cdot \exp \left\{-\sum_{j=1}^{n} \frac{\lambda}{2\left(s_{j}-s_{j-1}\right)}\left\|\vec{w}_{j}-\vec{w}_{j-1}\right\|^{2}\right. \\
& \left.\quad-\frac{\lambda}{2\left(T-s_{n}\right)}\left\|\vec{w}_{n}-\vec{\eta}\right\|^{2}\right\} d \vec{w}_{1} \ldots d \vec{w}_{n} d \vec{s}
\end{aligned}
$$


where $\Delta_{n}(T)=\left\{\vec{s}=\left(s_{1}, \ldots, s_{n}\right): 0<s_{1}<s_{2}<\cdots<s_{n}<T\right\}$, $s_{0}=0$ and $\vec{w}_{0}=\vec{\xi}$.

Proof. For notational purposes let $G_{\lambda}(\vec{\xi}, \vec{\eta})$ denote

$$
E\left(F\left(\lambda^{-1 / 2} \vec{x}+\vec{\xi}\right) \mid X\left(\lambda^{-1 / 2} \vec{x}+\vec{\xi}\right)\right)(\vec{\eta})
$$

Then

$$
\begin{aligned}
& G_{\lambda}(\vec{\xi}, \vec{\eta})=E\left[\sum_{n=0}^{\infty} \frac{1}{n !}\left[\int_{0}^{T} \theta\left(s, \lambda^{-1 / 2} \vec{x}(s)+\vec{\xi}\right) d s\right]^{n} \mid\right. \\
& \vec{x}(T)=\sqrt{\lambda}(\vec{\eta}-\vec{\xi})] \\
& =E\left[\sum_{n=0}^{\infty} \int_{\Delta_{n}(T)} \prod_{j=1}^{n} \theta\left(s_{j}, \lambda^{-1 / 2} \vec{x}\left(s_{j}\right)+\vec{\xi}\right) d \vec{s} \mid \vec{x}(T)=\sqrt{\lambda}(\vec{\eta}-\vec{\xi})\right] \\
& =\int_{C_{0}^{\nu}} \sum_{n=0}^{\infty} \int_{\Delta_{n}(T)} \prod_{j=1}^{n} \theta\left(s_{j}, \lambda^{-1 / 2} \vec{x}\left(s_{j}\right)+\vec{\xi}\right. \\
& \left.-\frac{s_{j}}{T}\left(\lambda^{-1 / 2} \vec{x}(T)+\vec{\xi}\right)+\frac{s_{j}}{T} \vec{\eta}\right) d \vec{s} m(d \vec{x}) \\
& =\sum_{n=0}^{\infty} \int_{\Delta_{n}(T)} \int_{C_{0}^{\nu}} \prod_{j=1}^{n} \theta\left(s_{j}, \lambda^{-1 / 2} \vec{x}\left(s_{j}\right)+\vec{\xi}\right. \\
& \left.-\frac{s_{j}}{T}\left(\lambda^{-1 / 2} \vec{x}(T)+\vec{\xi}\right)+\frac{s_{j}}{T} \vec{\eta}\right) m(d \vec{x}) d \vec{s} \\
& =\sum_{n=0}^{\infty} \int_{\Delta_{n}(T)}\left[(2 \pi)^{n+1} s_{1}\left(s_{2}-s_{1}\right) \cdots\left(T-s_{n}\right)\right]^{-\nu / 2} \\
& \int_{\mathbb{R}^{\nu(n+1)}} \exp \left\{-\sum_{j=1}^{n+1} \frac{\left\|\vec{u}_{j}-\vec{u}_{j-1}\right\|^{2}}{2\left(s_{j}-s_{j-1}\right)}\right\} \\
& \cdot \prod_{j=1}^{n} \theta\left(s_{j}, \lambda^{-1 / 2} \vec{u}_{j}+\vec{\xi}-\frac{s_{j}}{T}\left(\lambda^{-1 / 2} \vec{u}_{n+1}+\vec{\xi}\right)+\frac{s_{j}}{T} \vec{\eta}\right)
\end{aligned}
$$


with $s_{0}=0$ and $\vec{u}_{0}=\overrightarrow{0}$. Next let $\vec{w}_{0}=\vec{\xi}$,

$$
\vec{w}_{j}=\lambda^{-1 / 2} \vec{u}_{j}+\vec{\xi}-\frac{s_{j}}{T}\left(\lambda^{-1 / 2} \vec{u}_{n+1}+\vec{\xi}-\vec{\eta}\right) \quad \text { for } j=1, \ldots, n
$$

and let $\vec{w}_{n+1}=\lambda^{-1 / 2} \vec{u}_{n+1}+\vec{\xi}$. Then

$$
\begin{aligned}
G_{\lambda}(\vec{\xi}, \vec{\eta})= & \sum_{n=0}^{\infty} \int_{\Delta_{n}(T)}\left[\frac{\lambda^{n+1}}{(2 \pi)^{n+1} s_{1}\left(s_{2}-s_{1}\right) \cdots\left(T-s_{n}\right)}\right]^{\nu / 2} \\
& \cdot \int_{\mathbb{R}^{n \nu}}\left[\prod_{j=1}^{n} \theta\left(s_{j}, \vec{w}_{j}\right)\right] \\
& \cdot \exp \left\{-\sum_{j=1}^{n} \frac{\lambda\left\|\vec{w}_{j}-\vec{w}_{j-1}\right\|^{2}}{2\left(s_{j}-s_{j-1}\right)}\right\} \\
& \cdot\left[\int _ { \mathbb { R } ^ { \nu } } \operatorname { e x p } \left\{-\frac{\lambda}{T}\left\langle\vec{w}_{n+1}-\vec{\eta}, \vec{w}_{n}-\vec{\xi}\right\rangle-\frac{\lambda s_{n}}{2 T^{2}}\left\|\vec{w}_{n+1}-\vec{\eta}\right\|^{2}\right.\right. \\
& \left.\left.-\frac{\lambda}{2\left(T-s_{n}\right)}\left\|\vec{w}_{n+1}-\vec{w}_{n}-\frac{s_{n}}{T}\left(\vec{w}_{n+1}-\vec{\eta}\right)\right\|^{2}\right\} d \vec{w}_{n+1}\right] \\
& \quad \cdot d \vec{w}_{1} \cdots d \vec{w}_{n} d \vec{s} .
\end{aligned}
$$

Next carrying out the integration with respect to $\vec{w}_{n+1}$ in the above expression, simplifying, and multiplying both sides of the resulting expression by

$$
\left[\frac{\lambda}{2 \pi T}\right]^{\nu / 2} \exp \left\{-\frac{\lambda}{2 T}\|\vec{\eta}-\vec{\xi}\|^{2}\right\}
$$

we obtain equation (4.1) which concludes the proof of Theorem 4.1.

Recall that in equation (3.3), for $F \in S(\nu)$, we expressed the conditional Feynman integral $E^{\mathrm{anf}_{q}(F \mid X)(\vec{\xi})(\vec{\eta})}$ in terms of an integral over the infinite dimensional space $L_{2}^{\nu}[0, T]$. In our next theorem, as an application of Theorem 4.1, we obtain a series expansion of $E^{\operatorname{anf}_{q}}(F \mid X)$ in terms of integrals over finite dimensional spaces.

THEOREM 4.2. Let $F(\vec{x})=\exp \left\{\int_{0}^{T} \theta(s, \vec{x}(s)) d s\right\}$ with

$$
\theta(s, \vec{w})=\int_{\mathbb{R}^{\nu}} \exp \{i\langle\vec{w}, \vec{v}\rangle\} d \mu_{s}(\vec{v})
$$


where $\left\{\mu_{s}: 0 \leq s \leq T\right\}$ is a family from $M\left(\mathbb{R}^{\nu}\right)$ such that $\left\|\mu_{s}\right\| \in$ $L_{1}[0, T]$ and for each Borel set $B$ from $\mathbb{R}^{\nu}, \mu_{S}(B)$ is Borel measurable in $s$. Then for all real $q \neq 0$,

(4.3) $\quad E^{\operatorname{anf}_{q}(F \mid X)(\vec{\xi})(\vec{\eta})}$

$$
\begin{aligned}
& =\sum_{n=0}^{\infty} \int_{\Delta_{n}(T)} \int_{\mathbb{R}^{n \nu}} \exp \left\{-\frac{i}{2 q} \sum_{l=1}^{n} \sum_{j=1}^{l}\left(2-\delta_{j l}\right) s_{j}\left\langle\vec{v}_{j}, \vec{v}_{l}\right\rangle\right. \\
& +i \sum_{j=1}^{n}\left\langle\vec{\xi}, \vec{v}_{j}\right\rangle-\frac{i}{T} \sum_{j=1}^{n}\left\langle\vec{\xi}-\vec{\eta}, s_{j} \vec{v}_{j}\right\rangle \\
& \left.+\frac{i}{2 q T}\left\|\sum_{j=1}^{n} s_{j} \vec{v}_{j}\right\|^{2}\right\} \\
& \cdot d \mu_{S_{1}}\left(\vec{v}_{1}\right) \cdots d \mu_{S_{n}}\left(\vec{v}_{n}\right) d \vec{s}
\end{aligned}
$$

where $\delta_{j l}$ is the Kronecker delta.

Proof. We first note that $F(\vec{x})$ is Borel measurable [24, Corollary 3.2] and belongs to $S(\nu)$ [24, Remark 3.3]. Next using (4.1) and (4.2) we see that for

$$
\begin{array}{rl}
\lambda>0 & E\left(F\left(\lambda^{-1 / 2} \vec{x}+\vec{\xi}\right) \mid X\left(\lambda^{-1 / 2} \vec{x}+\vec{\xi}\right)\right)(\vec{\eta}) \\
= & {\left[\frac{2 \pi T}{\lambda}\right]^{\nu / 2} \exp \left\{\frac{\lambda}{2 T}\|\vec{\eta}-\vec{\xi}\|^{2}\right\}} \\
& \cdot \sum_{n=0}^{\infty} \int_{\Delta_{n}(T)}\left[\frac{\lambda^{n+1}}{(2 \pi)^{n+1} s_{1}\left(s_{2}-s_{1}\right) \cdots\left(T-s_{n}\right)}\right]^{\nu / 2} \\
& \cdot \int_{\mathbb{R}^{n \nu}}\left[\int_{\mathbb{R}^{n \nu}} \exp \left\{i \sum_{j=1}^{n}\left\langle\vec{w}_{j}, \vec{v}_{j}\right\rangle\right\} d \mu_{S_{1}}\left(\vec{v}_{1}\right) \cdots d \mu_{S_{n}}\left(\vec{v}_{n}\right)\right] \\
& \cdot \exp \left\{-\sum_{j=1}^{n} \frac{\lambda\left(s_{j}-s_{j-1}\right)}{2 \vec{w}_{j}-\vec{w}_{j-1} \|^{2}} \quad-\frac{\lambda}{2\left(T-s_{n}\right)}\left\|\vec{w}_{n}-\vec{\eta}\right\|^{2}\right\} d \vec{w}_{1} \cdots d \vec{w}_{n} d \vec{s} .
\end{array}
$$


Then using the Fubini Theorem and the formula (see equation (2.3))

$$
\begin{aligned}
\exp & \left\{-\frac{\lambda}{2\left(T-s_{n}\right)}\left\|\vec{w}_{n}-\vec{\eta}\right\|^{2}\right\} \\
& =\left[\frac{T-s_{n}}{2 \pi \lambda}\right]^{\nu / 2} \int_{\mathbb{R}^{\nu}} \exp \left\{i\left\langle\vec{u}, \vec{w}_{n}-\vec{\eta}\right\rangle-\frac{T-s_{n}}{2 \lambda}\|\vec{u}\|^{2}\right\} d \vec{u}
\end{aligned}
$$

we obtain

$$
\begin{aligned}
E\left(F\left(\lambda^{-1 / 2} \vec{x}+\vec{\xi}\right) \mid X\left(\lambda^{-1 / 2} \vec{x}+\vec{\xi}\right)\right)(\vec{\eta}) & \\
= & {\left[\frac{T}{2 \pi \lambda}\right]^{\nu / 2} \exp \left\{\frac{\lambda}{2 T}\|\vec{\eta}-\vec{\xi}\|^{2}\right\} } \\
& \cdot \sum_{n=0}^{\infty} \int_{\Delta_{n}(T)}\left[\frac{\lambda^{n}}{(2 \pi)^{n} s_{1}\left(s_{2}-s_{1}\right) \cdots\left(s_{n}-s_{n-1}\right)}\right]^{\nu / 2} \\
& \cdot \int_{\mathbb{R}^{(n+1) \nu}} \int_{\mathbb{R}^{n \nu}} \exp \left\{i \sum_{j=1}^{n}\left\langle\vec{w}_{j}, \vec{v}_{j}\right\rangle\right. \\
& -\sum_{j=1}^{n} \frac{\lambda}{2\left(s_{j}-s_{j-1}\right)}\left\|\vec{w}_{j}-\vec{w}_{j-1}\right\|^{2}+i\left\langle\vec{u}, \vec{w}_{n}-\vec{\eta}\right\rangle \\
\cdot d \vec{w}_{n} \cdots d \vec{w}_{1} d \vec{u} d \mu_{S_{1}}\left(\vec{v}_{1}\right) \cdots d \mu_{S_{n}}\left(\vec{v}_{n}\right) d \vec{s} . & \left.-\frac{T-s_{n}}{2 \lambda}\|\vec{u}\|^{2}\right\}
\end{aligned}
$$

Next we carry out the integration with respect to $\vec{w}_{n}, \vec{w}_{n-1}, \ldots$, $\vec{w}_{1}$ using the formula

$$
\begin{aligned}
& {\left[\frac{\lambda}{2 \pi\left(s_{j}-s_{j-1}\right)}\right]^{\nu / 2} \int_{\mathbb{R}^{\nu}} \exp \left\{-\frac{\lambda}{2\left(s_{j}-s_{j-1}\right)}\left\|\vec{w}_{j}-\vec{w}_{j-1}\right\|^{2}\right.} \\
& \left.+i\left\langle\vec{w}_{j}, \vec{u}+\vec{v}_{n}+\cdots+\vec{v}_{j}\right\rangle\right\} d \vec{w}_{j} \\
& =\exp \left\{i\left\langle\vec{w}_{j-1}, \vec{u}+\vec{v}_{n}+\cdot+\vec{v}_{j}\right\rangle-\frac{s_{j}-s_{j-1}}{2 \lambda}\left\|\vec{u}+\vec{v}_{n}+\cdots+\vec{v}_{j}\right\|^{2}\right\}
\end{aligned}
$$


successively for $j=n, n-1, \cdots, 1$ to obtain

$$
\begin{aligned}
& E\left(F\left(\lambda^{-1 / 2} \vec{x}+\vec{\xi}\right) \mid X\left(\lambda^{-1 / 2} \vec{x}+\vec{\xi}\right)\right)(\vec{\eta}) \\
& =\left[\frac{T}{2 \pi \lambda}\right]^{\nu / 2} \exp \left\{\frac{\lambda}{2 T}\|\vec{\eta}-\vec{\xi}\|^{2}\right\} \\
& \cdot \sum_{n=0}^{\infty} \int_{\Delta_{n}(T)} \int_{\mathbb{R}^{\nu} n}\left[\int_{\mathbb{R}^{\nu}} \exp \{-i\langle\vec{u}, \vec{\eta}\rangle\right. \\
& +i\left\langle\vec{\xi}, \vec{u}+\sum_{j=1}^{n} \vec{v}_{j}\right\rangle-\frac{T-s_{n}}{2 \lambda}\|\vec{u}\|^{2} \\
& \left.\left.-\sum_{j=1}^{n} \frac{\left(s_{j}-s_{j-1}\right)}{2 \lambda}\left\|\vec{u}+\vec{v}_{n}+\cdots+\vec{v}_{j}\right\|^{2}\right\} d \vec{u}\right] \\
& \cdot d \mu_{s_{1}}\left(\vec{v}_{1}\right) \cdots d \mu_{s_{n}}\left(\vec{v}_{n}\right) d \vec{s} \\
& =\left[\frac{T}{2 \pi \lambda}\right]^{\nu / 2} \exp \left\{\frac{\lambda}{2 T}\|\vec{\eta}-\vec{\xi}\|^{2}\right\} \\
& \cdot \sum_{n=0}^{\infty} \int_{\Delta_{n}(T)} \int_{\mathbb{R}^{\nu n}} \exp \left\{i\left\langle\vec{\xi}, \sum_{j=1}^{n} \vec{v}_{j}\right\rangle\right. \\
& \left.-\sum_{j=1}^{n} \frac{\left(s_{j}-s_{j-1}\right)}{2 \lambda}\left\|\vec{v}_{n}+\cdots+\vec{v}_{j}\right\|^{2}\right\} \\
& \cdot\left[\int_{\mathbb{R}^{\nu}} \exp \left\{-\frac{T}{2 \lambda}\|\vec{u}\|^{2} i\langle\vec{u}, \vec{\eta}-\vec{\xi}\rangle-\frac{1}{\lambda}\left\langle\vec{u}, \sum_{j=1}^{n} s_{j} \vec{v}_{j}\right\rangle\right\} d \vec{u}\right] \\
& \cdot d \mu_{S_{1}}\left(\vec{v}_{1}\right) \cdots d \mu_{s_{n}}\left(\vec{v}_{n}\right) d \vec{s} \text {. }
\end{aligned}
$$

But

$$
\begin{aligned}
\int_{\mathbb{R}^{\nu}} \exp \left\{-\frac{T}{2 \lambda}\|\vec{u}\|^{2}-i\langle\vec{u}, \vec{\eta}-\vec{\xi}\rangle-\frac{1}{\lambda}\left\langle\vec{u}, \sum_{j=1}^{n} s_{j} \vec{v}_{j}\right\rangle\right\} d \vec{u} \\
=\left[\frac{2 \pi \lambda}{T}\right]^{\nu / 2} \exp \left\{-\frac{\lambda}{2 T}\|\vec{\eta}-\vec{\xi}\|^{2}\right. \\
\left.+\frac{1}{2 \lambda T}\left\|\sum_{j=1}^{n} s_{j} \vec{v}_{j}\right\|^{2}-\frac{i}{T}\left\langle\vec{\xi}-\vec{\eta}, \sum_{j=1}^{n} s_{j} \vec{v}_{j}\right\rangle\right\} .
\end{aligned}
$$


Hence

$$
\begin{aligned}
& \text { (4.4) } E\left(F\left(\lambda^{-1 / 2} \vec{x}+\vec{\xi}\right) \mid X\left(\lambda^{-1 / 2} \vec{x}+\vec{\xi}\right)\right)(\vec{\eta}) \\
& =\sum_{n=0}^{\infty} \int_{\Delta_{n}(T)} \int_{\mathbb{R}^{n \nu}} \exp \left\{-\sum_{j=1}^{n} \frac{\left(s_{j}-s_{j-1}\right)}{2 \lambda}\left\|\vec{\xi}_{v_{n}}+\cdots+\vec{v}_{j}\right\|^{2}\right. \\
& +i\left\langle\vec{\xi}, \sum_{j=1}^{n} \vec{v}_{j}\right\rangle+\frac{1}{2 \lambda T}\left\|\sum_{j=1}^{n} s_{j} \vec{v}_{j}\right\|^{2} \\
& \left.-\frac{i}{T}\left\langle\vec{\xi}-\vec{\eta}, \sum_{j=1}^{n} s_{j} \vec{v}_{j}\right\rangle\right\} \\
& \cdot d \mu_{s_{1}}\left(\vec{v}_{1}\right) \cdots d \mu_{s_{n}}\left(\vec{v}_{n}\right) d \vec{s} \\
& =\sum_{n=0}^{\infty} \int_{\Delta_{n}(T)} \int_{\mathbb{R}^{n \nu}} \exp \left\{-\frac{1}{2 \lambda} \sum_{l=1}^{n} \sum_{j=1}^{l}\left(2-\delta_{j l}\right) s_{j}\left\langle\vec{v}_{j}, \vec{v}_{l}\right\rangle+i\left\langle\vec{\xi}, \sum_{j=1}^{n} \vec{v}_{j}\right\rangle\right. \\
& \left.-\frac{i}{T}\left\langle\vec{\xi}-\vec{\eta}, \sum_{j=1}^{n} s_{j} \vec{v}_{j}\right\rangle+\frac{1}{2 \lambda T}\left\|\sum_{j=1}^{n} s_{j} \vec{v}_{j}\right\|^{2}\right\} \\
& \cdot d \mu_{s_{1}}\left(\vec{v}_{1}\right) \cdots d \mu_{s_{n}}\left(\vec{v}_{n}\right) d \vec{s} .
\end{aligned}
$$

Since $F \in S(\nu)$, we know by Theorem 3.1 that the left-hand side of (4.4) has an analytic extension to $\mathbf{C}_{+}$and is continuous on $\mathbf{C}_{+}^{\sim}$. We will show that the same is true for the right-hand side of (4.4). We first show that the series converges absolutely for all $\vec{\xi}, \vec{\eta}$ in $\mathbb{R}^{\nu}$ and all $\lambda \in \mathbb{C}_{+}^{\sim}$. This follows from the fact that

$$
\sum_{l=1}^{n} \sum_{j=1}^{l}\left(2-\delta_{j l}\right)\left\langle\vec{v}_{j}, \vec{v}_{l}\right\rangle s_{j}-\frac{1}{T}\left\|\sum_{j=1}^{n} s_{j} \vec{v}_{j}\right\|^{2} \geq 0
$$

since

$$
\begin{array}{r}
\sum_{n=0}^{\infty} \int_{\Delta_{n}(T)} \int_{\mathbb{R}^{n \nu}} \mid \exp \left\{-\frac{1}{2 \lambda} \sum_{l=1}^{n} \sum_{j=1}^{l}\left(2-\delta_{j l}\right) s_{j}\left\langle\vec{v}_{j}, \vec{v}_{l}\right\rangle+i\left\langle\vec{\xi}, \sum_{j=1}^{n} \vec{v}_{j}\right\rangle\right. \\
\left.-\frac{i}{T}\left\langle\vec{\xi}-\vec{\eta}, \sum_{j=1}^{n} s_{j} \vec{v}_{j}\right\rangle+\frac{1}{2 \lambda T}\left\|\sum_{j=1}^{n} s_{j} \vec{v}_{j}\right\|^{2}\right\} \mid \\
\cdot d \mu_{s_{1}}\left(\vec{v}_{1}\right) \cdots d \mu_{s_{n}}\left(\vec{v}_{n}\right) d \vec{s}
\end{array}
$$


(continued)

$$
\begin{aligned}
& \leq \sum_{n=0}^{\infty} \int_{\Delta_{n}(T)} \int_{\mathbf{R}^{n \nu}} \exp \left\{-\frac{1}{2} \operatorname{Re}\left[\frac{1}{\lambda}\right] \sum_{l=1}^{n} \sum_{j=1}^{l}\left(2-\delta_{j l}\right) s_{j}\left\langle\vec{v}_{j}, \vec{v}_{l}\right\rangle\right. \\
& \left.+\frac{1}{2 T} \operatorname{Re}\left[\frac{1}{\lambda}\right]\left\|\sum_{j=1}^{n} s_{j} \vec{v}_{j}\right\|^{2}\right\} \\
& \cdot d \mu_{s_{1}}\left(\vec{v}_{1}\right) \cdots d \mu_{s_{n}}\left(\vec{v}_{n}\right) d \vec{s} \\
& \leq \sum_{n=0}^{\infty} \int_{\Delta_{n}(T)} \int_{\mathbb{R}^{n \nu}} d \mu_{s_{1}}\left(\vec{v}_{1}\right) \cdots d \mu_{s_{n}}\left(\vec{v}_{n}\right) d \vec{s} \\
& =\sum_{n=0}^{\infty} \frac{1}{n !} \int_{[0, T]^{n}}\left[\prod_{j=1}^{n}\left\|\mu_{s_{j}}\right\|\right] d \vec{s}=\sum_{n=0}^{\infty} \frac{1}{n !}\left[\int_{0}^{T}\left\|\mu_{s}\right\| d s\right]^{n} \\
& =\exp \left\{\int_{0}^{T}\left\|\mu_{s}\right\| d s\right\}<\infty .
\end{aligned}
$$

Thus using Morea's Theorem and the Dominated Convergence Theorem we obtain that the right-hand side of (4.4) is an analytic function of $\lambda$ throughout $\mathbb{C}_{+}$and is continuous in $\lambda$ on $\mathbb{C}_{+}^{\sim}$. Thus (4.3) is established which completes the proof of Theorem 4.2.

The following corollary is immediate using Theorem 4.2 in conjunction with Theorem 3.2.

Corollary 4.1. Let $F$ be as in Theorem 4.2. Then the conclusions of Theorem 3.2 hold and for $\psi \in L_{1}\left(\mathbb{R}^{\nu}\right), J_{q}^{\mathrm{an}}(F) \psi$ is given by (3.6) (and (1.1)) with $E^{\mathrm{anf}_{q}(F \mid X)}$ given by (4.3).

5. The $L_{1} \rightarrow L_{\infty}$ theory. In this section, as in $[4,10,19]$ we restrict our attention to the case $\nu=1$ since [20, section 6] Johnson and Skoug gave counterexamples showing that the $L_{1}\left(\mathbb{R}^{\nu}\right) \rightarrow L_{\infty}\left(\mathbb{R}^{\nu}\right)$ theory doesn't hold for $\nu>1$. In $[4,19]$ an $\mathscr{L}\left(L_{1}(\mathbb{R}), L_{\infty}(\mathbb{R})\right)$ theory of the operator-valued Feynman integral $J_{q}^{\mathrm{an}}(F)$ was developed for functions of the form

$$
F(x)=\exp \left\{\int_{0}^{T} \theta(s, x(s)) d s\right\}
$$

with appropriate assumptions on $\theta$; the most general being as follows: Let $r \in(2, \infty]$ and let $\theta:[0, T] \times \mathbb{R} \rightarrow \mathbf{C}$ be a Borel measurable 
function such that for a.e. $s$ in $[0, T], \theta(s, \cdot)$ is in $L_{1}(\mathbb{R})$ with $L_{1}$ norm $\|\theta(s, \cdot)\|_{1}$ in $L_{r}[0, T]$. In this section we will show that for such $F, J_{q}^{\text {an }}(F)$ is given by the formula

$$
\begin{aligned}
& \left(J_{q}^{\mathrm{an}}(F) \psi\right)(\xi) \\
& =\int_{-\infty}^{\infty} E^{\operatorname{anf}_{q}}(F \mid X)(\xi)(\eta)\left[\frac{q}{2 \pi i T}\right]^{1 / 2} \exp \left\{\frac{q i}{2 T}(\eta-\xi)^{2}\right\} \psi(\eta) d \eta
\end{aligned}
$$

for $\psi \in L_{1}(\mathbb{R})$.

REMARK. Note that $F$ of the form (5.1) may be unbounded and thus not in $S(1)$ and hence Theorem 3.2 and Corollary 4.1 do not apply to $F$ given by (5.1) with $\theta$ as above.

THEOREM 5.1. Let $F$ be given by (5.1) with $\theta$ as above and let $X(y)=y(T)$ for $y \in C[0, T]$. Then for all real $q \neq 0$,

$$
\begin{aligned}
E^{\operatorname{anf}_{q}}(F \mid X)(\xi)(\eta)\left[\frac{q}{2 \pi i T}\right]^{1 / 2} \exp \left\{\frac{i q}{2 T}(\eta-\xi)^{2}\right\} \\
=\sum_{n=0}^{\infty}\left[\frac{-i q}{2 T}\right]^{(n+1) / 2} \\
\quad \cdot \int_{\Delta_{n}(T)}\left[s_{1}\left(s_{2}-s_{1}\right) \cdots\left(s_{n}-s_{n-1}\right)\left(T-s_{n}\right)\right]^{-1 / 2} \\
\quad \int_{\mathbb{R}^{n}}\left[\prod_{j=1}^{n} \theta\left(s_{j}, w_{j}\right)\right] \\
\quad \cdot \exp \left\{\sum_{j=1}^{n} \frac{i q}{2\left(s_{j}-s_{j-1}\right)}\left(w_{j}-w_{J-1}\right)^{2}\right. \\
\left.+\frac{i q}{2\left(T-s_{n}\right)}\left(w_{n}-\eta\right)^{2}\right\} d w_{1} \cdots d w_{n} d \vec{s}
\end{aligned}
$$

where $\Delta_{n}(T)=\left\{\vec{s}=\left(s_{1}, \cdots, s_{n}\right): 0<s_{1}<s_{2}<\cdots<s_{n}<T\right\}, s_{0}=$

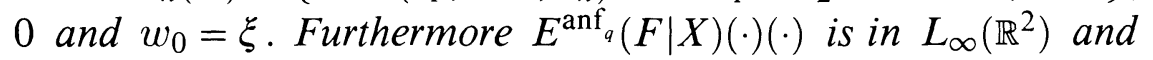
(5.4) $\| E^{\operatorname{anf}_{q}(F \mid X)(\cdot)(\cdot) \|_{\infty}}$

$$
\leq \sum_{n=0}^{\infty}\left|\frac{q}{2 \pi}\right|^{n / 2} \frac{T^{n(2-p) / 2 p}[\Gamma(1-p / 2)]^{(n+1) / p}\left[\int_{0}^{T}\|\theta(s, \cdot)\|_{1}^{r} d s\right]^{n / r}}{(n !)^{1 / r}\{\Gamma[(n+1)(1-p / 2)]\}^{1 / p}}
$$

where $\Gamma$ denotes the gamma function and $p$ is such that $1 / p+1 / r=1$. 
Proof. Using equation (4.1) with $\nu=1$ we see that for each $\lambda>0$,

(5.5) $E\left(F\left(\lambda^{-1 / 2} x+\xi\right) \mid X\left(\lambda^{-1 / 2} x+\zeta\right)\right)(\eta)\left[\frac{\lambda}{2 \pi T}\right]^{1 / 2}$

$$
\begin{aligned}
& \cdot \exp \left\{-\frac{\lambda}{2 T}(\eta-\zeta)^{2}\right\} \\
= & \sum_{n=0}^{\infty}\left[\frac{\lambda}{2 \pi}\right]^{(n+1) / 2} \\
& \cdot \int_{\Delta_{n}(T)}\left[s_{1}\left(s_{2}-s_{1}\right) \cdots\left(s_{n}-s_{n-1}\right)\left(T-s_{n}\right)\right]^{-1 / 2} \\
& \cdot \int_{\mathbf{R}^{n}}\left[\prod_{j=1}^{n} \theta\left(s_{j}, w_{j}\right)\right] \\
& \cdot \exp \left\{-\sum_{j=1}^{n} \frac{\lambda}{2\left(s_{j}-s_{j-1)}\right.}\left(w_{j}-w_{j-1}\right)^{2}\right. \\
& \left.-\frac{\lambda}{2\left(T-s_{n}\right)}\left(w_{n}-\eta\right)^{2}\right\} d w_{1} \cdots d w_{n} d \vec{s} .
\end{aligned}
$$

For notational purposes let $H_{\lambda}(\xi, \eta)$ denote the right-hand side of (5.5). Then for all $(\lambda, \xi, \eta) \in \mathbf{C}_{+}^{\sim} \times \mathbb{R} \times \mathbb{R}$ we see that

$$
\begin{aligned}
\left|H_{\lambda}(\xi, \eta)\right| \leq & \sum_{n=0}^{\infty}\left[\frac{|\lambda|}{2 \pi}\right]^{(n+1) / 2} \\
& \cdot \int_{\Delta_{n}(T)}\left[s_{1}\left(s_{2}-s_{1}\right) \cdots\left(s_{n}-s_{n-1}\right)\left(T-s_{n}\right)\right]^{-1 / 2} \\
& \cdot \int_{\mathbb{R}^{n}} \prod_{j=1}^{n}\left|\theta\left(s_{j}, w_{j}\right)\right| d w_{1} \cdots d w_{n} d \vec{s} \\
\leq & \sum_{n=0}^{\infty}\left[\frac{|\lambda|}{2 \pi}{ }^{(n+1) / 2}\right] \cdot \int_{\Delta_{n}(T)}\left[s_{1}\left(s_{2}-s_{1}\right) \cdots\left(s_{n}-s_{n-1}\right)\left(T-s_{n}\right)\right]^{-1 / 2} \\
& \cdot\left[\prod_{j=1}^{n}\left\|\theta\left(s_{j}, \cdot\right)\right\|_{1}\right] d \vec{s}
\end{aligned}
$$




$$
\begin{aligned}
\leq & \sum_{n=0}^{\infty}\left[\frac{|\lambda|}{2 \pi}^{(n+1) / 2}\right] \\
& \cdot\left\{\int_{\Delta_{n}(T)}\left[s_{1}\left(s_{2}-s_{1}\right) \cdots\left(s_{n}-s_{n-1}\right)\left(T-s_{n}\right)\right]^{-p / 2} d \vec{s}\right\}^{1 / p} \\
& \cdot\left\{\int_{\Delta_{n}(T)} \prod_{j=1}^{n}\left\|\theta\left(s_{j}, \cdot\right)\right\|_{1}^{r} d \vec{s}\right\}^{1 / r} .
\end{aligned}
$$

But

$$
\begin{aligned}
\int_{\Delta_{n}(T)} \prod_{j=1}^{n}\left\|\theta\left(s_{j}, \cdot\right)\right\|_{1}^{r} d \vec{s} & =\frac{1}{n !} \int_{0}^{T} \cdots \int_{0}^{T} \prod_{j=1}^{n}\left\|\theta\left(s_{j}, \cdot\right)\right\|_{1}^{r} d s_{1} \cdots d s_{n} \\
& =\frac{1}{n !}\left[\int_{0}^{T}\left\|\theta\left(s_{j}, \cdot\right)\right\|_{1}^{r} d s\right]^{n}
\end{aligned}
$$

and as was shown in $[19$, p. 652],

$$
\begin{gathered}
\int_{\Delta_{n}(T)}\left[s_{1}\left(s_{2}-s_{1}\right) \cdots\left(s_{n}-s_{n-1}\right)\left(T-s_{n}\right)\right]^{-p / 2} d \vec{s} \\
=\frac{T^{-p / 2} T^{n(2-p) / 2}[\Gamma(1-p / 2)]^{n+1}}{\Gamma[(n+1)(1-p / 2)]} .
\end{gathered}
$$

Thus for all $(\lambda, \xi, \eta) \in \mathbb{C}_{+}^{\sim} \times \mathbb{R} \times \mathbb{R}$,

(5.6) $\left|H_{\lambda}(\xi, \eta)\right|$

$$
\begin{aligned}
\leq & \sum_{n=0}^{\infty}\left[\frac{|\lambda|}{2 \pi}\right]^{(n+1) / 2} \\
& \frac{\left.T^{n(2-p) / 2 p}[\Gamma(1-p / 2)]^{(n+1) / p}\left[\int_{0}^{T}\|\theta(s, \cdot)\|_{1}^{r} d s\right)\right]^{n / r}}{(n !)^{1 / r}\{\Gamma[(n+1)(1-p / 2)]\}^{1 / p} T^{1 / 2}} .
\end{aligned}
$$

But since for large positive $w$,

$$
\frac{1}{\Gamma(w)}<\frac{2 e^{w} \sqrt{w}}{\sqrt{2 \pi} w^{w}}
$$

it is not hard to see that the series on the right-hand side of (5.6) converges for each $\lambda \in \mathbb{C}_{+}^{\sim}$; in fact uniformly on compact subsets of 
$\mathbb{C}_{+}$. Thus the right-hand side of $(5.5)$ is an analytic function of $\lambda$ on $\mathbb{C}_{+}$and continuous on $\mathbb{C}_{+}^{\sim}$ which establishes $(5.3)$. The inequality (5.4) follows easily from (5.6) and (5.3).

TheOREM 5.2. Let $F$ and $X$ be as in Theorem 5.1. Then for all real $q \neq 0$, the analytic operator-valued Feynman integral $J_{a}^{\text {an }}(F)$ exists as an element of $\mathscr{L}\left(L_{1}(\mathbb{R}), L_{\infty}(\mathbb{R})\right)$ and for each $\psi \in L_{1}(\mathbb{R})$ is given by (5.2).

Proof. By [19] we know that $J_{q}^{\text {an }}(F)$ exists as an element of $\mathscr{L}\left(L_{1}(\mathbb{R}), L_{\infty}(\mathbb{R})\right.$ ) (actually as an element of $\mathscr{L}\left(L_{1}(\mathbb{R}), C_{0}(\mathbb{R})\right.$ ). We need to establish equation (5.2) with $E^{\operatorname{anf}_{q}}(F \mid X)(\xi)(\eta)$ given by (5.3). But, proceeding as in the beginning of the proof of Theorem 3.2, we see that for all $\lambda>0$

$$
\begin{aligned}
\left(I_{\lambda}(F) \psi\right)(\xi)= & \int_{-\infty}^{\infty} E\left(F\left(\lambda^{-1 / 2} x+\xi\right) \mid X\left(\lambda^{-1 / 2} x+\xi\right)\right)(\eta) \psi(\eta) \\
& \cdot\left[\frac{\lambda}{2 \pi T}\right]^{1 / 2} \exp \left\{-\frac{\lambda}{2 T}(\eta-\xi)^{2}\right\} d \eta
\end{aligned}
$$

where $E\left(F\left(\lambda^{-1 / 2} x+\xi\right) \mid X\left(\lambda^{-1 / 2} x+\xi\right)\right)(\eta)$ is given by (4.1) with $\nu=1$. But, as was shown in Theorem 5.1, $E\left(F\left(\lambda^{-1 / 2} x+\xi\right) \mid X\left(\lambda^{-1 / 2} x+\xi\right)\right)(\eta)$ is an analytic function of $\lambda$ throughout $\mathbb{C}_{+}$and so

$$
\begin{aligned}
& \left(I_{\lambda}^{\mathrm{an}}(F) \psi\right)(\xi) \\
& =\int_{-\infty}^{\infty} E^{\mathrm{anw}_{\lambda}(F \mid X)(\xi)(\eta)}\left[\frac{\lambda}{2 \pi T}\right]^{1 / 2} \\
& \cdot \exp \left\{-\frac{\lambda}{2 T}(\eta-\xi)^{2}\right\} \psi(\eta) d \eta
\end{aligned}
$$

for all $\lambda \in \mathbb{C}_{+}$. Taking the limit of both sides of (5.7) as $\lambda \rightarrow-i q, \lambda \in$ $\mathbb{C}_{+}$, establishes (5.2).

\section{REFERENCES}

[1] R. H. Cameron and D. A. Storvick, An operator valued function space integral and a related integral equation, J. Math. and Mech., 18 (1968), 517-552.

[2] __ An integral equation related to the Schroedinger equation with application to integration in function space, Problems in Analysis, Princeton Univ. Press, Princeton, (1970), 175-193. 
[3] R. H. Cameron and D. A. Storvick, An operator valued function space integral applied to integrals of functions of class $L_{2}$, J. Math. Anal. Appl., 42 (1973), 330-372.

[4] _ An operator valued function space integral applied to integrals of functions of class $L_{1}$, Proc. London Math. Soc., 27 (1973), 345-360.

[5] _ Some Banach algebras of analytic Feynman integrable functionals, in Analytic Functions, Kozubnik, 1979, Springer Lecture Notes in Mathematics, Berlin, 798 (1980), 18-67.

[6] Analytic Feynman integral solutions of an integral equation related to the Schroedinger equation, J. Analyse Math., 30 (1980), 34-66.

[7] _ A new translation theorem for the analytic Feynman integral, Rev. Roumaine Math. Pure Appl., 27 (1982), 937-944.

[8] _ A simple definition of the Feynman integral, with applications, Memoirs of the Amer. Math. Soc., No. 288, 46 (1983), 1-46.

[9] __ New existence theorems and evaluation formulas for sequential Feynman integrals, Proc. London Math. Soc., 52 (1986), 557-581.

[10] J. S. Chang, Stability theorems for the Feynman integral: the $\mathscr{L}\left(L_{1}(\mathbb{R}), C_{0}(\mathbb{R})\right)$ Theory, Supplemento ai Rendiconti del Circolo Mathematico di Palermo, serie II, numero 17 (1987), 135-151.

[11] K. S. Chang, Scale-invariant measurability in Yeh Wiener space, J. Korean Math. Soc., 19 (1982), 61-67.

[12] K. S. Chang and J. S. Chang, Evaluation of some conditional Wiener integrals, Bulletin Korean Math. Soc., 21 (1984), 99-106.

[13] K. S. Chang, G. W. Johnson and D. L. Skoug, Functions in the Banach algebra $S(\nu)$, J. Korean Math. Soc., 24 (1987), 151-158.

[14] D. M. Chung and S. J. Kang, Conditional Wiener integrals and an integral equation, J. Korean Math. Soc., 25 (1988), 37-52.

[15] D. M. Chung and D. L. Skoug, Conditional analytic Feynman integrals and a related Schroedinger integral equation, to appear in SIAM J. Math. Anal.

[16] G. W. Johnson and M. L. Lapidus, Generalized Dyson series, generalized Feynman diagrams, the Feynman integral and Feynman's operational calculus, Mem. Amer. Math. Soc., 62, No. 351 (1986).

[17] G. W. Johnson and D. L. Skoug, Operator-valued Feynman integrals of finitedimensional functionals, Pacific J. Math., 34 (1970), 774-780.

[18] __ A Banach algebra of Feynman integrable functions with application to an integral equation formally equivalent to Schoredinger's equation, J. Funct. Anal., 12 (1973), 129-152.

[19] - The Cameron-Storvick function space integral: The $L_{1}$ theory, J. Math. Anal. and Appl., 50 (1975), 647-667.

[20] - The Cameron-Storvick function space integral: an $\mathscr{L}\left(L_{p}, L_{p^{\prime}}\right)$ theory, Nagoya Math. J., 60 (1976), 93-137.

[21] __ Scale-invariant measurability in Wiener space, Pacific J. Math., 83 (1979), 157-176.

[22] __ Notes on the Feynman integral, I, Pacific J. Math., 93 (1981), 313-324.

[23] _ Notes on the Feynman integral, II, J. Funct. Anal., 41 (1981), 277-289.

[24] _ Notes on the Feynman integral III: The Schoredinger equation, Pacific J. Math., 105 (1983), 321-358.

[25] _ Stability theorems for the Feynman integral, Supplemento ai Rendiconti del Circolo Matematico di Palermo, 8 (1985), 361-377.

[26] C. Park and D. L. Skoug, The Feynman integral of quadratic potentials depending on $n$ time parameters, Nagoya Math. J., 110 (1988), 151-162. 
[27] C. Park and D. L. Skoug, A simple formula for conditional Wiener integrals with applications, Pacific J. Math., 135 (1988), 381-394.

[28] J. Yeh, Inversion of conditional expectations, Pacific J. Math., 52 (1974), 63 i640.

[29] _ Inversion of conditional Wiener integrals, Pacific J. Math., 59 (1975), 623-638.

[30] - Transformation of conditional Wiener integrals under translation and the Cameron-Martin translation theorem, Tohoku Math. J., 30 (1978), 505-515.

Received November 16, 1988. Research of the first author was partially supported by the BSRIP, and the Ministry of Education, Korea (1988).

SOGANG UNIVERSITY

SEOUL 121, KOREA

AND

Miami University

OXFORD, OH 45056

AND

UNIVERSITY OF NEBRASKA

LINCOLN, NE 68588-0323 


\section{PACIFIC JOURNAL OF MATHEMATICS EDITORS}

\author{
V. S. VARADARAJAN \\ (Managing Editor) \\ University of California \\ Los Angeles, CA 90024-1555-05 \\ Herbert Clemens \\ University of Utah \\ Salt Lake City, UT 84112 \\ Thomas ENRIGHT \\ University of California, San Diego \\ La Jolla, CA 92093
}

\section{R. FINN}

Stanford University

Stanford, CA 94305

Hermann FlaschKa

University of Arizona

Tucson, AZ 85721

VAUGHAN F. R. Jones

University of California

Berkeley, CA 94720

STEVEN KeRCKHOFF

Stanford University

Stanford, CA 94305
C. C. MOORE

University of California

Berkeley, CA 94720

Martin ScharlemanN

University of California

Santa Barbara, CA 93106

HAROLd STARK

University of California, San Diego

La Jolla, CA 92093

\section{ASSOCIATE EDITORS}
R. ARENS
E. F. BECKENBACH
B. H. NeumanN
F. Wolf
K. YoshidA (1906-1982)
(1904-1989)

\section{SUPPORTING INSTITUTIONS}
UNIVERSITY OF ARIZONA
UNIVERSITY OF BRITISH COLUMBIA
CALIFORNIA INSTITUTE OF TECHNOLOGY
UNIVERSITY OF CALIFORNIA
MONTANA STATE UNIVERSITY
UNIVERSITY OF NEVADA, RENO
NEW MEXICO STATE UNIVERSITY
OREGON STATE UNIVERSITY
UNIVERSITY OF OREGON
UNIVERSITY OF SOUTHERN CALIFORNIA
STANFORD UNIVERSITY
UNIVERSITY OF HAWAII
UNIVERSITY OF TOKYO
UNIVERSITY OF UTAH
WASHINGTON STATE UNIVERSITY
UNIVERSITY OF WASHINGTON 


\section{Pacific Journal of Mathematics}

Vol. 146, No. $1 \quad$ November, 1990

Primo Brandi and Anna Salvadori, A quasi-additivity type condition and

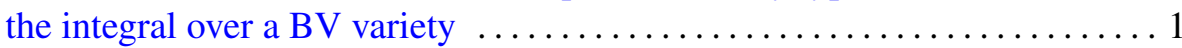

Dong M. Chung, Chull Park and David Lee Skoug, Operator-valued

Feynman integrals via conditional Feynman integrals ..............21

Paul Jolissaint, Index for pairs of finite von Neumann algebras . .........43

Miodrag Mateljević and Miroslav Pavlović, Multipliers of $H^{p}$ and

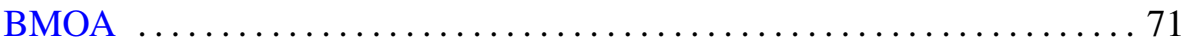

Himadri Kumar Mukerjee, Poincaré cobordism exact sequences and

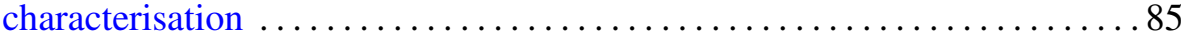

Thomas H. Otway, The coupled Yang-Mills-Dirac equations for differential

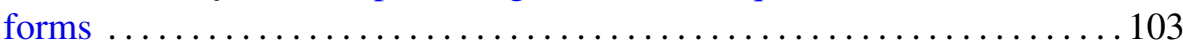

Sechiko Takahashi, Nevanlinna parametrizations for the extended

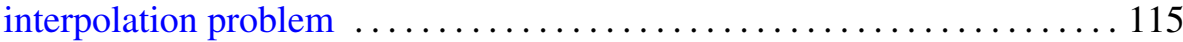

P. C. Trombi, Uniform asymptotics for real reductive Lie groups ........ 131 